\title{
Infektionen bei Blutspendern
}

\section{Zusammenfassung}

Die Verhinderung von transfusionsassoziierten Infektionen stellt eine der größten Herausforderungen der Transfusionsmedizin dar. Die Kombination von konsequenter Spenderauswahl und strikter Testung jeder Blutspende auf Infektionserreger mit validierten Testverfahren konnte das Übertragungsrisiko deutlich verringern. Durch die systematische Auswertung von infektionsepidemiologischen Daten können neue Risikogruppen identifiziert, neue Infektionen und unbekannte Infektionswege erkannt werden. Die engmaschige Surveillance von Blutspendern im Hinblick auf HIV-, Hepatitis-C-Virus-, Hepatitis-B-Virus- und Syphilisinfektionen, die gemäß § 22 Transfusionsgesetz vom Robert Koch-Institut (RKI) durchgeführt wird, trägt zur Aufrechterhaltung des sehr hohen Sicherheitsstandards bei Transfusionen in Deutschland bei. Die hierbei gewonnenen Daten helfen, Kriterien für die Spenderselektion anzupassen und die Notwendigkeit von etablierten und neuen Testverfahren zu prüfen. Darüber hinaus ermöglicht die Surveillance eine Abschätzung des verbleibenden Infektionsrisikos bei Bluttransfusionen. Über die bekannten transfusionsrelevanten Infektionen hinaus müssen zunehmend auch andere Infektionskrankheiten, beispielsweise West-Nil-Virusinfektionen oder die variante CreutzfeldtJakob-Erkrankung (vCJK), im Hinblick auf ihre Transfusionsrelevanz infektionsepidemiologisch bewertet werden.

\section{Schlüsselwörter}

Blutspender · Infektionen - Surveillance · Transfusionssicherheit

\section{Historische Entwicklung}

Jede Infektion, bei der Erreger im Blut zirkulieren, kann grundsätzlich durch Bluttransfusionen übertragen werden. Bereits in den Jahren 1911 und 1915 wurden die ersten Berichte über Malaria- und Syphiliserkrankungen im Zusammenhang mit Transfusionen veröffentlicht. Eine 1943 publizierte Arbeit beschrieb das gehäufte Auftreten von Gelbsucht 3-4 Monate nach einer Transfusion [1]. Diese Beobachtungen führten bereits in der ersten Hälfte des 20. Jahrhunderts zur Formulierung von verbindlichen Vorgaben für die Auswahl von Blutspendern. Diese wurden 1940 in den „Richtlinien für die Einrichtung des Blutspendewesens im Deutschen Reich“ festgelegt [2]. Im Jahr 1968 veröffentlichte das Bundesgesundheitsamt erstmals für die gesamte Bundesrepublik Deutschland gültige Richtlinien für das Blutspendewesen, in denen auch Kriterien für die Spenderauswahl festgelegt wurden [3]. Der Fortschritt der Infektiologie ermöglichte die Entwicklung von Maßnahmen, welche die Infektionssicherheit von Transfusionen sukzessive verbesserten. Noch vor der Entwicklung spezifischer Testverfahren für die einzelnen Krankheitserreger wurden Surrogatmarker wie die Messung von Leberenzymen, z.B. der Alanin-Aminotransferase (ALT), eingeführt. Die ALT ist auch heute noch Bestandteil des Blutspendescreenings. Als erster erregerspezifischer Test wurde ein Suchtest auf Lues eingesetzt. Nach Entdeckung des Hepatitis-B-Virus (HBV) folgte 1970 die verbindliche Prüfung der Spender auf Hepatitis-B-Oberflächenantigen (HbsAg) bei jeder Spende. Hiermit konnte die Zahl der durch Transfusionen verursachten Hepatitis-B-Virusinfektionen deutlich verringert werden. Durch die Einführung des Screenings auf Antikörper gegen das Hepatitis-C-Virus (AntiHCV) im Jahr 1990 konnten auch die bis dahin häufigen Übertragungen von $\mathrm{He}$ patitis $\mathrm{C}$ durch Transfusionen reduziert werden. Durch die Einführung des HCVGenomnachweises 1999 wurde dieses Risiko noch weiter stark vermindert. Bereits vor der Entdeckung des für AIDS ursächlichen Erregers, HIV, Anfang der 8oer-Jahre erkannte man aufgrund epidemiologischer Zusammenhänge, dass diese Immunschwäche auch transfusionsassoziiert auftreten konnte [4]. Diese Erkenntnis führte zu einer Modifikation der Spenderauswahlkriterien, um die Produktsicherheit weiter zu erhöhen. Die Testung der Blutspender auf Antikörper gegen HIV (Anti-HIV) wurde im Laufe des Jahres 1985 zur Pflicht und hat dazu beigetragen, dass das Risiko, eine HIV-Infektion durch Blut und Blutprodukte in Deutschland zu erwerben, verschwindend klein geworden ist.

Die Kriterien für die Auswahl der Blutspender und die Austestung der Blutspenden gewährleisten heute zusammen mit den Kontrollinstanzen für die Blutspendedienste und der infektionsserologischen Überwachung der Spender die Sicherheit der Blutprodukte in Deutschland. Die einzelnen Maßnahmen werden im Folgenden kurz erläutert.

\footnotetext{
(C) Springer-Verlag 2003

Dr.R.Offergeld

Abteilung für Infektionsepidemiologie,

Robert Koch-Institut, Nordufer 20, 13353 Berlin

E-Mail:OffergeldR@RKI.de
} 
Bundesgesundheitsbl - Gesundheitsforsch Gesundheitsschutz 2003 -46:775-779

DOI 10.1007/s00103-003-0678-2

\section{R. Offergeld $\cdot$ K. Stark $\cdot$ 0. Hamouda}

\section{Infections associated with blood transfusion}

\begin{abstract}
Avoiding transfusion-associated infections is one of the greatest challenges for transfusion medicine. The combination of consequent donor selection and state of the art testing of every donation has substantially reduced the risk of transfusion-associated infections. Infectious disease surveillance provides information on new and emerging infections and can identify new sources of infection. Close surveillance of the blood donor population with regard to HIV, hepatitis $C$, hepatitis $B$ and syphilis infections, as warranted by $\S 22$ of the Transfusion Act by the Robert Koch-Institute, helps to maintain the high degree of transfusion safety in Germany. Criteria for donor selection and testing can subsequently be modified. Infectious disease surveillance in the blood donor population is also necessary in order to calculate the current residual risk of transfusion-transmitted infections. Apart from the known transfusion-relevant infections, emerging infections such as West Nile virus and variant Creutzfeldt-Jakob disease have to be epidemiologically evaluated with regard to transfusion medicine.
\end{abstract}

\section{Keywords}

Blood donor · Infections - Surveillance · Transfusion safety

\section{Originalien und Übersichtsarbeiten}

\section{Spenderauswahlkriterien}

Die Sicherheit von nicht inaktivierbaren Blutprodukten hängt entscheidend von der Häufigkeit des Auftretens von Infektionen im Kollektiv der Blutspender ab. Daher wurden Kriterien entwickelt, um Personen mit bestehenden Infektionen oder deutlich erhöhten Infektionsrisiken auf Dauer oder zeitlich begrenzt von der Spende auszuschließen. Die Spenderausschluss- und Rückstellkriterien werden in den aktuellen „Richtlinien zur Gewinnung von Blut und Blutbestandteilen und zur Anwendung von Blutprodukten (Hämotherapie)“ der Bundesärztekammer und des Paul-Ehrlich-Instituts (PEI) festgelegt [5]. Sie berücksichtigen sowohl nachgewiesene Infektionen, die durch Transfusionen übertragen werden können, als auch Risikofaktoren für den Erwerb von Infektionen. Unter anderem wurden Regelungen hinsichtlich der Infektionen mit HIV, HBV, HCV und dem Erreger der Syphilis sowie für tropische Infektionen festgelegt. Spender, die an einer transfusionsrelevanten Infektion leiden oder ein gegenüber der Allgemeinbevölkerung stark erhöhtes Risiko für den Erwerb einer solchen haben, werden dauerhaft von der Blutspende ausgeschlossen. Diejenigen Spender, die sich kurzfristig, z. B. durch eine Reise, einem Risiko für den Erwerb einer Infektion ausgesetzt haben, werden zeitlich befristet von der Blutspende zurückgestellt. Die Rückstellung erfolgt, um eine Übertragung in der Frühphase der Infektion zu verhindern, wenn der Spender noch keine Krankheitssymptome aufweist und der Erreger durch Testverfahren noch nicht sicher nachgewiesen werden kann.

\section{Spender, die an einer trans- fusionsrelevanten Infektion leiden, werden dauerhaft von der Blutspende ausgeschlossen}

Darüber hinaus kann das Paul-EhrlichInstitut als zuständige Bundesoberbehörde durch Auflagen im Rahmen von Stufenplanverfahren zusätzliche Spenderausschlusskriterien oder Testungen anordnen. Ein Beispiel hierfür, das aufgrund der theoretisch möglichen Gefahr für die Übertragung der neuen Variante der Creutzfeldt-Jakob-Erkrankung (vCJK) durch Blutprodukte angeordnet wurde, ist der Ausschluss von Personen, die sich zwischen 1980 und 1996 kumulativ länger als 6 Monate in Großbritannien oder Nordirland aufgehalten haben. Für diese Personen bestand potenziell ein höheres BSE-Expositionsrisiko über die Nahrungskette.

\section{Spenderscreening}

In den Hämotherapie-Richtlinien werden auch die erforderlichen Mindestanforderungen für die Testung von Blutprodukten festgelegt. Derzeit sind in Deutschland die Testung auf HIV-1/2Antikörper, HCV-Antikörper, HCV-Genom, HbsAg, Antikörper gegen Treponema pallidum sowie die Bestimmung der ALT zwingend vorgeschrieben. Ein großer Teil der Blutspendeeinrichtungen führt auf freiwilliger Basis weitere Tests durch. Hierzu zählen z. B. Genomnachweise für HIV und HBV oder ein Test auf Antikörper gegen das HepatitisB-Core-Antigen (Anti-HBc). Ab dem 1.5.2004 wird der HIV-Genomnachweis ebenfalls als Pflichttest vor der Freigabe eines zellulären Blutproduktes oder von gefrorenem Frischplasma eingeführt.

\section{Kontrolle der Blutspendedienste}

Die Blutspendeeinrichtungen unterliegen Kontrollinstanzen, die in entsprechenden Gesetzen, insbesondere im Arzneimittelund Transfusionsgesetz (TFG) festgelegt sind $[6,7]$. So erfolgt die Zulassung der im Blutspendedienst hergestellten Produkte nach entsprechender Prüfung durch das PEI. Die Herstellungserlaubnis als pharmazeutischer Unternehmer wird durch die zuständigen Behörden der Bundesländer erteilt. Diese führen unter Beteiligung von Experten aus dem PEI wiederum regelmäßig Inspektionen der Blutspendedienste durch. Der Blutspendedienst selbst hat gemäß $₫ 1$ a der Betriebsverordnung für pharmazeutische Unternehmer [8] ein System zur Qualitätssicherung vorzuhalten. Dem Robert Koch-Institut kommt in diesem Netzwerk die Aufgabe der bundesweiten epidemiologischen Überwachung (Surveillance) von Infektionen bei Blutspendern zu. Gemäß dieser Aufgabe werden Infektionsdaten nach $\$ 22$ TFG an das RKI gemeldet und dort ausgewertet. Eine weitere Einrichtung, die zur Gewährleistung der Blutsicherheit in Deutschland beiträgt, ist der Arbeitskreis Blut (AK Blut), ein Expertengremium nach $\$ 24$ Transfu- 
sionsgesetz. Er beobachtet und bewertet die Weiterentwicklung im Bereich Transfusionsmedizin und verabschiedet Voten und Stellungnahmen zu transfusionsrelevanten Themen und berät die Bundesregierung in Fragen der Sicherheit bei der Gewinnung und Anwendung von Blut und Blutprodukten.

\section{Infektionsepidemiologische Surveillance}

Die infektionsepidemiologische Surveillance von Blutspendern leistet einen wichtigen Beitrag zur Sicherheit von Blutprodukten, da hierdurch neue Infektionsrisiken identifiziert und bekannte neu bewertet werden können. Die Analyse dieser Daten trägt zur Festlegung neuer Spenderauswahlkriterien bei und bildet eine Grundlage für die Entscheidung über die Einführung weiterer Testverfahren. Darüber hinaus ermöglicht sie die Erhebung von aufschlussreichen epidemiologischen Daten einer definierten Subpopulation der Gesamtbevölkerung. Umfassende Untersuchungen zur Infektionsepidemiologie bei Blutspendern wurden in Deutschland, insbesondere im Hinblick auf HIV-Infektionen seit 1985 durchgeführt $[10,11,12]$. Seit 1994 wurden Daten für HIV-, HCV- und HBV-Infektionen, bei Blutspendern im Rahmen einer gemeinsamen Studie des Berufsverbandes der Transfusionsmediziner und des RKI erhoben und ausgewertet $[13,14,15]$. Mit dem $\$ 22$ TFG wurden die Meldungen gesetzlich verankert. Ihre Erfassung und Auswertung obliegt seit Mitte 1998 allein dem RKI. Art und Umfang der Meldungen wurden in der Verordnung über das Meldewesen vom Dezember 2001 und im Votum 22 des AK Blut vom November 1999 weiter präzisiert [16]. Danach werden folgende Daten vierteljährlich von jedem Blutspendedienst erhoben: Anzahl der Spender für die unterschiedlichen Spendearten (Vollblut, Plasmapherese, Zytapherese), aufgeschlüsselt nach Spendertyp (Erstspendewilliger/Erstspender/Mehrfach-spender), Geschlecht und Altersgruppe sowie durchschnittlichem Spendeintervall. Zusätzlich wird die Gesamtzahl der Spender, die bestätigt positiv für HIV, HCV, HBV oder den Erreger der Syphilis getestet wurden, aufgelistet. Von bestätigt positiven Spendern werden folgende Angaben anonymisiert dokumentiert: demografische Angaben, individuelles Spendeverhalten, Informationen zum Infektionsmodus und die detaillierten Laborergebnisse zu HIV-, HCV-, HBVInfektionen und zur ALT.

Die Meldungen müssen von allen Blutspendediensten in Deutschland gemacht werden. Träger dieser Einrichtungen sind das Deutsche Rote Kreuz, staatlich-kommunale Blutspendedienste, private Blutspendedienste, Blutspendeeinrichtungen der Bundeswehr und industrielle Plasmaphereseeinrichtungen. Nachdem die ersten Studien zur Prävalenz und Inzidenz von HIV-Infektionen noch auf Datenerhebungen in einzelnen Spendediensten beruhten, lag der Anteil der meldenden Einrichtungen seit Beginn der bundesweiten Studie im Jahr 1995 immer deutlich über 90\%. Im Jahr 2001 konnten erstmals von $100 \%$ der Blutspendeeinrichtungen Daten erhoben werden.

\section{Ergebnisse 2001}

Im Jahr 2001 wurden infektionsserologische Untersuchungen bei 547.436 Erstspendern ohne infektionsserologische Voruntersuchung und bei Erstspendewilligen sowie bei 5.159.332 Spenden von Mehrfachspendern durchgeführt [17]. Vom Gesamtaufkommen der differenzierbaren Spenden entfielen $81 \%$ auf Vollblutspenden, $15 \%$ auf Plasmapheresespenden und 3\% auf Zytapheresespenden. Gemäß Votum 22 des AK Blut wurde eine Infektion als bestätigt angesehen, wenn ein auffälliger Screeningtest durch einen positiven Bestätigungstest ergänzt wurde und/ oder ein Genomnachweistest reproduzierbar positiv war. Die kumulativen Infektionsraten für alle Spendenarten des Jahres 2001 sind in der Tabelle 1 dargestellt.

Bei der Auswertung kann für die Erstspender und die Erstspendewilligen die Prävalenz der Infektion ermittelt werden. Da jedoch bei den Wiederholungsspendern aus technischen Gründen bislang nur die Zahl der Spenden übermittelt wurde, lässt sich anhand von durch- schnittlichen Spendeintervallen die Zahl der Spender, die diese Spenden leisten, nur näherungsweise schätzen. Aus Stichprobenuntersuchungen lässt sich ableiten, dass der durchschnittliche Vollblutspender im Mittel 2 Spenden im Jahr leistet [14]. Nur die genaue Erhebung von Spenderzahlen würde es ermöglichen, eine exakte Inzidenz für die Infektionen $\mathrm{zu}$ ermitteln. Dies soll die Datenerhebung, wie im TFG vorgesehen, zukünftig berücksichtigen.

Bei der Analyse zeigt sich, dass der Anteil der Infektionen bei den Erstspenden um den Faktor 10 bis 100 höher war als bei den Mehrfachspenden. Diese Daten sind vergleichbar mit denen der zuvor untersuchten Zeiträume von 1996-1999 [18] und den Ergebnissen internationaler Studien aus anderen Industrieländern [19]. Personen, die sich erstmals in einem Blutspendedienst vorstellen (Erstspender und Erstspendewillige), werden durch $\mathrm{Ge}$ spräch und Informationsblätter auf die Risiken und möglichen Infektionswege von transfusionsrelevanten Infektionen hingewiesen. Dennoch werden einige Infektionserkrankungen erst bei der Blutspende diagnostiziert. Mehrfachspender stellen hingegen sowohl aufgrund ihrer wiederholten Testung als auch durch ihre gezielte Auswahl ein hoch selektiertes Kollektiv dar, bei dem die Infektionsrisiken im Vergleich zur Allgemeinbevölkerung wesentlich geringer sind.

Bei den Syphilisinfektionen ist zu beachten, dass die aktuellen Meldekriterien nicht zwischen einer akuten und einer abgelaufenen Infektion unterscheiden, sodass bei der Prävalenz auch lange zurückliegende, ausgeheilte Infektionen in die Berechnung eingehen. Da Treponema pallidum in Erythrozytenkonzentraten nur begrenzt überlebensfähig ist, spielen transfusionsassoziierte Syphiliserkrankungen im Vergleich zu den viralen Erkrankungen heute eine deutlich geringere Rolle [20]. Die fortlaufende Beobach-

$\begin{aligned} & \text { Tabelle } 1 \\
& \text { Infektionsraten für HIV, HCV, HBV und Syphilis, alle Spenden } 2001\end{aligned}$
\begin{tabular}{lcc} 
Infektion & $\begin{array}{l}\text { Prävalenz bei Erstspendern/ } \\
\text { Erstspendewilligen } \\
\text { (Infektionen/100.000 Spenden) }\end{array}$ & $\begin{array}{l}\text { Serokonversionen } \\
\text { bei Mehrfachspendern } \\
\text { (Infektionen/100.000 Spenden) }\end{array}$ \\
\hline HIV & 4,57 & 0,54 \\
HCV & 92,43 & 1,63 \\
HBV & 155,45 & 1,43 \\
Syphilis & 32,52 & 1,55
\end{tabular}


tung der Serokonversionen für Syphilis ist jedoch, insbesondere unter Beachtung der weltweiten Zunahme der Erkrankung, besonders wichtig, um auch in Deutschland eine Trenderfassung außerhalb der Risikogruppen vornehmen zu können [21].

\section{In Deutschland sind trans- fusionsrelevante Infektionen bei Blutspendern selten}

Vergleicht man die Prävalenzdaten der Hepatitisinfektionen mit denen einer repräsentativen Bevölkerungsstichprobe aus dem Bundes-Gesundheitssurvey von 1998, so war bei den Blutspendern die HCV-Prävalenz im Jahr 2001 erfreulicherweise um den Faktor 4 niedriger als in der untersuchten Stichprobe der Allgemeinbevölkerung [22]. Für die HBV-Infektionen ist der direkte Vergleich der Daten aufgrund unterschiedlicher diagnostischer Kriterien zwar erschwert, dennoch ist die Prävalenz der HBV-Infektion im Kollektiv der Blutspender ebenfalls um den Faktor 4 niedriger als in der untersuchten Bevölkerungsstichprobe.

Für HIV-Infektionen liegen keine Untersuchungen zur bundesweiten Prävalenz vor. Die Daten der anonymen Untersuchung Neugeborener (Anonymous unlinked testing, AUT) können jedoch zum Vergleich herangezogen werden. In dieser Untersuchung, die zwischen 1993 und 1997 durchgeführt wurde, wurde das Blut Neugeborener auf das Vorliegen einer HIV-Infektion untersucht. Aufgrund der Plazentagängigkeit der HIV-Antikörper konnte auf diese Weise bei 95\% der gebärenden Frauen in Berlin und Niedersachsen die Infektionsrate im Beobachtungszeitraum erfasst werden. Sie kann als Näherungswert für die HIV-Prävalenz in der weiblichen, sexuell aktiven Bevölkerung gesehen werden [23]. Die Prävalenz in diesem Kollektiv lag ebenfalls, abhängig vom Wohnort, um den Faktor 2-15 höher als im Kollektiv der Blutspender. Diese Ergebnisse bestätigen die Effektivität und Sorgfalt der Spenderauswahl der einzelnen Blutspendedienste. Die vergleichende Analyse der Meldedaten mit den Erhebungen nach dem Infektionsschutzgesetz (IfSG) [24] wird wegen der größeren Datenbasis zukünftig von großem Interesse sein und zu einem besseren Erkennen neuer Risikogruppen beitragen.

Vergleicht man die Ergebnisse der infektionsepidemiologischen Erhebun- gen in Deutschland von 1996-2001 kumulativ über alle Spendenarten, so erkennt man eine unverändert niedrige Prävalenz der HIV- und HBV-Infektionen und eine gleich bleibend niedrige Serokonversionsrate für beide Infektionen unter Blutspendern. Bei den HCV-Infektionen zeigt sich jedoch seit 1996 eine signifikante Abnahme sowohl der Prävalenz (von 143 auf 92 Infektionen/100.00o Spender) als auch der Rate an Serokonversionen (von 7,3 auf 1,6 Infektionen/ 100.000 Spenden). Einen vergleichbaren Trend zeigen Studien aus den USA und Frankreich [19, 25, 26]. Da sich im Beobachtungszeitraum die Spenderauswahlkriterien im Hinblick auf HCV nicht verändert haben, bleibt die Ursache für diese Abnahme der HCV-Infektionen in der Population der Blutspender offen. Ob diesem Trend ein Rückgang der HCV-Infektionen in der Gesamtbevölkerung zugrunde liegt, wird zukünftig die Analyse der nach IfSG gewonnenen Daten zeigen.

\section{Restrisiko bei Transfusionen}

Sowohl für den Patienten als auch für den behandelnden Arzt ist wichtig einzuschätzen, welche Infektionsrisiken mit der Anwendung von Blutprodukten verbunden sind. Das Restrisiko, durch eine Transfusion infiziert zu werden, resultiert hauptsächlich aus der Möglichkeit, dass eine Spende im so genannten ,di-agnostischen Fenster" geleistet wurde. Dies bedeutet, dass eine Infektion in ihrer Frühphase durch die angewandten Testverfahren noch nicht entdeckt wurde, der Spender jedoch bereits infektiös war. Die Übertragung einer Infektion durch Blutprodukte hängt zusätzlich von weiteren Faktoren ab: Auf der Spenderseite ist die Prävalenz der Infektion in der Population der Blutspender, die Symptomatik der infizierten Personen und die Dauer der Infektiosität entscheidend. Auf der Produktseite ist wichtig, wie viele Krankheitserreger im Endprodukt vorhanden sind, ob diese überlebensfähig sind und ob wirksame Inaktivierungsverfahren zum Einsatz kommen. Schließlich kommt es auf der Empfängerseite darauf an, ob der Patient gegen die Infektion immun ist. Risiken, die durch neue Erregervarianten, chronisch seronegative, aber infizierte Spender oder Testversagen entstehen, werden dagegen als vernachlässigbar gering eingestuft. Da die Infektionswahrscheinlichkeit mit den „klassischen“ transfusionsrelevanten Erregern (HIV, HCV, HBV und Treponema pallidum) durch Bluttransfusionen nur noch sehr gering ist, sind direkte prospektive Erhebungen zum Auftreten von Infektionen unter Transfusionsempfängern nicht geeignet, eine Risikoabschätzung durchzuführen. Die sehr geringe Anzahl an Infektionen würde voraussetzen, dass eine extrem hohe Zahl an Empfängern von Blutprodukten untersucht werden müsste, um eine statistisch signifikante Aussage zu erlauben. Daher wurden zur Risikoabschätzung mathematische Modelle entwickelt, mit deren Hilfe die Wahrscheinlichkeit geschätzt werden kann, dass eine unerkannt infektiöse Spende geleistet wird. Das gängigste Modell bezieht die Inzidenz der Infektion in der Spenderpopulation sowie die Zeitspanne des diagnostischen Fensters in die Kalkulation ein [27, 28, 29]. Für Deutschland wurde 1998 das Restrisiko für HIV-Infektionen auf 1:1.000.000, für HCV-Infektionen auf 1:100.000 und für HBV-Infektionen auf 1:200.000 [14] geschätzt. Durch die Einführung des Genomnachweises für HCV wurde das diagnostische Fenster weiter geschlossen, sodass das Risiko für transfusionsassoziierte HCV-Infektionen spürbar reduziert wurde und aktuell auf kleiner als 1:1.000.000 geschätzt wird. Der zusätzliche Sicherheitsgewinn durch die geplante Einführung des HIV-1-Genomnachweises als Pflichttest im Blutspendescreening sowie durch den freiwilligen HBV-Genomnachweis ist durch die relativ geringereVerkürzung des diagnostischen Fensters nicht so stark ausgeprägt wie bei HCV-Infektionen, kann jedoch - unter Berücksichtigung des aufgeführten mathematischen Modells - zur Entdeckung von weiteren bislang unerkannt infektiösen Spenden führen [27]. Der hohe Standard der angewandten Verfahren bedingt, dass jede weitere Reduktion des schon jetzt sehr kleinen Restrisikos einer transfusionsassoziierten Infektion durch die Einführung zusätzlicher Testverfahren mit einem hohen Kostenaufwand verbunden sein kann. Die Einschätzung des Restrisikos und die Bewertung der Erforderlichkeit von neuen und eingeführten Testverfahren muss daher auch eine KostenNutzen-Analyse einbeziehen.

Die Verdachtsfälle von transfusionsassoziierten Infektionen müssen demPaulEhrlich-Institut gemeldet werden. Durch das PEI sind bezogen auf einen Transfusionszeitpunkt seit 1995 insgesamt 4 trans- 
fusionsassoziierte HIV-Infektionen, 11 HBV-Infektionen und 24 HCV-Infektionen als wahrscheinlich bewertet worden. Sämtliche HCV-Transmissionen erfolgten vor der Einführung des HCV-Genomnachweises im Jahr 1999, was den zusätzlichen Sicherheitsgewinn dieser Maßnahme eindrucksvoll demonstriert [30].

\section{Weitere transfusionsrelevante Erreger}

Wie dargestellt, konnte im Hinblick auf Infektionen mit HIV, HCV, HBV und mit dem Erreger der Syphilis eine sehr hohe Sicherheit im Transfusionswesen erreicht werden. Es ist aber bekannt, dass eine Vielzahl von weiteren Viren, Bakterien und Protozoen transfusionsassoziiert übertragen werden kann [31, 32, 33, 34]. Diese Erreger werden zurzeit nicht über die spezifische transfusionsmedizinische Surveillance erfasst. Wie wichtig diesbezügliche epidemiologische Erkenntnisse jedoch auch im Hinblick auf die Blutsicherheit sind, zeigt sich am Beispiel des West-Nil-Virus (WNV). Das WNV ist ein Flavivirus, das seit 1999 in den USA epidemisch auftritt und dort auch transfusionsassoziiert übertragen wurde $[35,36]$. Entsprechend hat die amerikanische $\mathrm{Zu}$ lassungsbehörde FDA veranlasst, dass ein zum Screening von Blutspendern geeigneter WNV-Genomnachweis entwickelt wurde, der seit Juli 2003 in den USA flächendeckend zum Einsatz kommt. Inwiefern WNV auch in Deutschland von Bedeutung ist, lässt sich gegenwärtig schwer einschätzen, da zurzeit epidemiologische Daten zur Prävalenz fehlen. Aktuell vom RKI, dem PEI und weiteren Instituten begonnene epidemiologische Studien werden es ermöglichen, das diesbezügliche transfusionsassoziierte Risiko und die Notwendigkeit von Testverfahren oder Spenderausschlusskriterien in Deutschland zu klären.

Für die Transfusionsmedizin ist weiterhin von Bedeutung, ob Erreger von transmissiblen spongiformen Enzephalopathien (TSE, z.B. vCJK) mit dem Blut übertragen werden können. In Tierversuchen konnte gezeigt werden, dass die Infektion durch das Blut natürlich infizierter auf gesunde Tiere übertragbar ist [37]. Grundsätzlich scheint demnach eine transfusionsassoziierte Infektion möglich $\mathrm{zu}$ sein. Da zurzeit Testverfahren fehlen, die sich zum Screening von Blutspendern eignen, wurden als erste präventive Maßnah- me diejenigen Personen von der Blutspende ausgeschlossen, die sich zwischen 1980 und 1996 kumulativ mehr als 6 Monate in Großbritannien und Nordirland aufgehalten haben und somit ein potenziell höheres Expositionsrisiko gegenüber BSE-kontaminiertem Rindfleisch hatten. Weitere präventive Maßnahmen wie der Ausschluss von Transfusionsempfängern von der Blutspende werden derzeit geprüft. Auch hier spielt bei der Bewertung der Maßnahmen, die zur Steigerung der Sicherheit eingesetzt werden, die epidemiologische Bewertung der Infektionen eine entscheidende Rolle. Dies gilt umso mehr, als dass zusätzliche Maßnahmen in der Regel mit Kosten und einer weiteren Einschränkung des Spenderkollektivs einhergehen. Um die sichere Versorgung Deutschlands mit Blutprodukten aufrechtzuerhalten, müssen diese verschiedenen Aspekte gegeneinander abgewogen werden.

\section{Fazit}

In Deutschland sind transfusionsrelevante Infektionen bei Blutspendern selten. Die sorgfältige Spenderauswahl und eine konsequente Testung aller Spenden gewährleisten den sehr hohen Sicherheitsstandard bei Bluttransfusionen. Die infektionsepidemiologische Surveillance leistet hierbei einen wichtigen Beitrag zum Erkennen neuer und alter Infektionsrisiken und hat bei der Modifikation von Sicherheitsmaßnahmen einen hohen Stellenwert. Neben der Gewährleistung des hohen Sicherheitsstandards auf Produktseite trägt auch die qualitätsgesicherte Anwendung von Blutkomponenten und Plasmaderivaten zur Vermeidung von Transfusionsrisiken bei.

\section{Literatur}

1. Beeson PB (1943) Jaundice occurring one to four months after transfusion of blood or plasma.JAMA 121:1332-1334

2. Runderlass des Reichsministers des Inneren, betr. Richtlinien für die Einrichtung des Blutspendewesens im Deutschen Reich (1940). Reichs-Gesundheitsblatt 17:328-336

3. Richtlinien mit Informationen zur Blutgruppenbestimmung und Bluttransfusion (1968). Dtsch Ärztebl 65:1987-1998

4. Ragni MV, Lewis JH, Spero JA, Bontempo FA (1983) Aquired-immunodeficiency-like syndrome in two haemophiliacs.Lancet 1(8318):213-214

5. Richtlinien zur Gewinnung von Blut und Blutbestandteilen und zur Anwendung von Blutprodukten (Hämotherapie) (2000). Bundesgesundheitsblatt 43:555-589

6. Gesetz über den Verkehr mit Arzneimitteln (Arzneimittelgesetz-AMG) vom 24.08.1976, Neufassung durch Bekanntmachung vom 11.12.1998. Bundesgesetzblatt Jahrgang 1998, Teil l:3586

7. Gesetz zur Regelung des Transfusionswesens (Transfusionsgesetz) vom 1.Juli 1998. Bundesgesetzblatt Jahrgang 1998, Teil l:1752
8. Betriebsverordnung für pharmazeutische Unternehmer (PharmaBetrV) vom 08.03.1985 (BGBI I,S 546) zuletzt geändert durch $\S 35$ des TFG vom 01.07.1998 (BGBI I,S 1752)

9. Empfehlung zum Meldewesen nach Transfusionsgesetz $\S$ 22 (2000) Votum 22 des Arbeitskreis Blut.

Bundesgesundheitsblatt 43:249-252

10. Maurer C, Kiehl W, Altmann D (1993) Zur HIV-Prävalenz und HIV-Inzidenz bei Blutspendern in Baden-Württemberg. Beitr Infusionsther 31:5-9

11. Köhler M,Mayr WR (1995) Nebenwirkungen der Therapie mit Blut und Blutprodukten. Hämostaseologie 15:192-196

12. Glück D, Koerner K, Caspari G, et al. (1987) Prävalenz von HIV-Antikörpern bei Blutspendern in der Bundesrepublik Deutschland und in West-Berlin. Dtsch Med Wochenschr 112:1603-1610

13. Glück D, Maurer C, Kubanek B (1997) Infektionsmarker bei Blutspenden. Infusionsther Transfusionsmed 24:167-170

14. Glück D, Kubanek B, Maurer C, Petersen N (1998) Seroconversion of $\mathrm{HIV}, \mathrm{HCV}$, and $\mathrm{HBV}$ in blood donors in 1996 risk of virus transmission by blood products in Germany. Infus Ther Trasfus Med 25:82-4

15. Glück D (1999) Risiko der HIV-, HCV- und HBV-Übertragung durch Blutpräparate. Infus Ther Transfus Med 26:335-338

16. Verordnung über das Meldewesen nach $\S \S 21$ und 22 des Transfusionsgesetzes (Transfusionsgesetz-Meldeverordnung - TFGMV) 2001. Bundesgesetzblatt 1:3737-3738

17. Offergeld R, Kramer M, Burger R, et al.(2002) Prevalence and incidence of HIV, HCV and HBV infections among German blood donors in 2001. Infus Ther Transfus Med 29(S1):22

18. Stark K, Werner E, Seeger E et al. (2002) Infections with HIV, $\mathrm{HBV}$, and HCV among blood donors in Germany 1998 and 1999. Infus Ther Transfus Med 29:305-307

19. Glynn SA, Kleinman SH, Schreiber GB, et al. (2000) Trends in incidence and prevalence of major transfusion-transmissible viral infections in US blood donors, 1991 to 1996. JAMA 284:229-235

20. Schmidt PJ (2001) Syphilis, a disease of direct transfusion. Transfusion 41:1069-1071

21. Aktuelle Entwicklung der Syphilis in Deutschland. Epid Bull (2002) 39:329-333

22. Thierfelder W, Meisel H, Schreier E, Dortschy R (1999) Die Prävalenz von Antikörpern gegen Hepatitis-A- HepatitisB- und Hepatitis-C-Viren in der deutschen Bevölkerung. Gesundheitswesen 61:110-114

23. Siedler A, Hamouda 0, Schwartländer B (1998) Anonymous unlinked testing as a sentinel approach:experience in Germany.J Epidemiol Comm Health 52 [Suppl 1]:25S-27S

24. Bales S, Baumann HG, Schnitzler N (2001) Infektionsschutzgesetz, Kommentar und Vorschriftensammlung. W. Kohlhammer, Berlin

25. Pillonel J, Laperche S, Saura C, et al. (2002) Trends in residual risk of transfusion transmitted viral infections in France between 1992 and 2000. Transfusion 42:980-988

26. Dodd RY, Notari IV EP, Stramer SL (2002) Current prevalence and incidence of infectious disease markers and estimated window-period risk in the American Red Cross blood donor population. Transfusion 42:975-979

27. Schreiber GB, Busch MP, Kleinman SH, Korelitz JJ (1996) The risk of transfusion-transmitted viral infections. NEJM 334:685-690

28. Glynn SA, Kleinman SH, Wright DJ, Busch MP (2002) International application of the incidence rate/window period model. Transfusion; 42:966-972

29. Korelitz J Busch MP, Kleinman SH et al. (1997) A method for estimating hepatitis $B$ virus incidence rates in volunteer blood donors. Transfusion; 37:634-640

30. Persönliche Mitteilung, Dr. A.Graul, Paul-Ehrlich-Institut, Langen

31. Montag T, Lange H, Schmidt U, et al. (1999) Bakterielle Kontamination von Blutprodukten. BundesgesundheitsblGesundheitsforsch-Gesundheitsschutz 42:132-142

32. Chamberland ME, Alter HJ, Busch MP, et al. (2001) Emerging infectious disease issues in blood safety. EID 7(s3):12-15

33. Gürtler LG (2002) Virus safety of human blood, plasma and derived products. Thrombosis Res 107:39-45

34. Busch MP, Kleinman SH, Nemo GJ (2003) Current and emerging infectious risks of blood transfusions. JAMA 289:959-962

35. Biggerstaff BJ, Petersen LR (2002) Estimated risk of West Nile virus transmission through blood transfusion during an epidemic in Queens, New York City. Transfusion 42:1019-1026

36. Update: Investigations of West Nile Virus infections in recipients of organ transplantation and blood transfusion (2002) MMWR 51:833-836

37. Hunter N, Foster J, Chong A, et al. (2002) Transmission of prion disease by blood transfusion. J Gen Virol 83:2897-2905 